Open Access

\title{
The effects of normovolemic anemia and blood transfusion on cerebral microcirculation after severe head injury
}

Judith Bellapart ${ }^{1,2^{*}}$ (D) Kylie Cuthbertson ${ }^{5}$, Kimble Dunster ${ }^{1,6}$, Sara Diab ${ }^{1,6}$, David G. Platts ${ }^{1,7}$, Christopher Raffel ${ }^{1,7}$, Levon Gabrielian ${ }^{8}$, Adrian Barnett ${ }^{1,9}$, Jennifer Paratz ${ }^{3,4}$, Rob Boots ${ }^{2}$ and John F. Fraser ${ }^{1,10}$

\author{
* Correspondence: \\ judithbellapart@gmail.com \\ ${ }^{1}$ Critical Care Research Group, \\ University of Queensland, Brisbane, \\ Queensland, Australia \\ ${ }^{2}$ Intensive Care Department, Royal \\ Brisbane and Women's Hospital, \\ Butterfield Street, Herston, QLD \\ 4025, Australia \\ Full list of author information is \\ available at the end of the article
}

\begin{abstract}
Background: Cerebral regional microcirculation is altered following severe head injury. In addition to tissue disruption, partial pressure of tissue oxygenation is impaired due to an increase in the oxygen tissue gradient. The heterogenic distribution of cerebral microcirculation is multifactorial, and acute anemia challenges further the delivery of oxygen to tissues. Currently, a restrictive transfusion threshold is globally applied; however, it is unclear how anemia modifies regional cerebral microcirculation; hence, it is unclear if by aiming to a global endpoint, specific anatomical regions undergo ischemia. This study aims to quantify the temporal changes in cerebral microcirculation after severe head injury, under the effect of anemia and transfusion. It also aims to assess its effects specifically at the ischemic penumbra compared to contralateral regions and its interactions with axonal integrity in real time. Twelve ovine models were subjected to a severe contusion and acceleration-deceleration injury. Normovolemic anemia to a restrictive threshold was maintained after injury, followed by autologous transfusion. Direct quantification of cerebral microcirculation used cytometric count of color-coded microspheres. Axonal injury was assessed using amyloid precursor protein staining.
\end{abstract}

Results: A mixed-effect regression model from pre-transfusion to post-transfusion times with a random intercept for each sheep was used. Cerebral microcirculation amongst subjects with normal intracranial pressure was maintained from baseline and increased further after transfusion. Subjects with high intracranial pressure had a consistent reduction of their microcirculation to ischemic thresholds $(20-30 \mathrm{ml} / 100 \mathrm{~g} / \mathrm{min})$ without an improvement after transfusion. Cerebral $\mathrm{PtiO}_{2}$ was reduced when exposed to anemia but increased in a 9.6-fold with transfusion 95\% Cl 5.6 to 13.6 ( $p$ value < 0.001 ).

Conclusions: After severe head injury, the exposure to normovolemic anemia to a restrictive transfusion threshold, leads to a consistent reduction on cerebral microcirculation below ischemic thresholds, independent of cerebral perfusion pressure. Amongst subjects with raised intracranial pressure, microcirculation does not improve after transfusion. Cerebral oxymetry is impaired during anemia with a statistically significant increase after transfusion. Current transfusion practices in neurocritical care are based on a rigid hemoglobin threshold, a view that excludes cerebral metabolic demands and specific needs. An RCT exploring these concepts is warranted.

Keywords: Anemia, APP staining, Histology, Microcirculation, Microspheres 


\section{Background}

Severe head injury often combines the effects of a contusion with an accelerationdeceleration force leading to axonal tearing, cytogenic, vasogenic edema, impaired cerebral autoregulation, and subsequent perfusion mismatch [1]. Secondary patho-physiologic processes may ultimately result in areas of ischemia and cerebral infarct. These changes are commonly found in regions where cerebral microcirculation (RMBF) has been critically reduced [2, 3]. An additional contributor to cerebral ischemia is partial pressure of tissue oxygenation $\left(\mathrm{PtiO}_{2}\right)$ with a series of studies focussing on the relevance of cerebral oximetry [4-7]. While current evidence-based practice attempts to minimise the use of allogenic blood transfusions, specifically amongst critically ill patients $[8,9]$ by establishing a restrictive transfusion threshold [10-13]; controversy still remains regarding the safety of these measures, specifically when facing sustained low $\mathrm{PtiO}_{2}$ levels, during the acute phase of head injury $[4,7,14-19]$. Studies have showed that a sustained hemoglobin concentration of $7 \mathrm{~g} / \mathrm{dL}$ (known as the "restrictive transfusion threshold") correlates with neurological injury due to cerebral tissue hypoxia [16]. In addition, blood transfusion has shown to significantly increase $\mathrm{PtiO}_{2}$ independently of cerebral perfusion pressure [17, 18]. Furthermore, questions continue to rise despite a recent randomised control study [20] not really challenging the proposed level of acceptable anemia at a hemoglobin of $7 \mathrm{~g} / \mathrm{dL}$. In the meantime, the absence of clinical equipoise as well as the lack of methodological feasibility in designing a study that focuses on cerebral RMBF during anemia and severe head injury, leads to the need of experimental models, to further challenge this question. In the absence of clinically available measures of cerebral microcirculation, physiological markers of cerebral ischemia and hypoperfusion rather than global metrics such as mortality outcomes may be more appropriate endpoints [21].

This study challenges the following hypothesis:

- Hypothesis one: RMBF after severe head injury is reduced over time, specifically, at the contusion regions when compared to contralateral regions.

- Hypothesis two: The maintenance of normovolemic anemia followed by self-transfusion, does not significantly impair RMBF.

- Hypothesis three: RMBF is most reduced in regions of severe tissue disruption, shown by a high amyloid precursor protein (APP) staining score.

- Hypothesis four: The maintenance of normovolemic anemia significantly reduces cerebral Pti02, these changes revert following self-transfusion.

The aims of this study are described as follows:

- First aim: To quantify the temporal changes in cerebral RMBF after severe head injury in an ovine model, specifically at the ischemic penumbra compared to contralateral regions, using cytometric counting of color-coded microspheres.

- Second aim: To simultaneously superimpose cerebral blood flow data with histopathology data, by using APP staining at each region of interest and at each time point before and after severe head injury.

- Third aim: To quantify cerebral Pti02 through all time-points to demonstrate its changes to anemia and transfusion. 


\section{Methods}

\section{Animal care and preparation}

Sheep were the most appropriate animal model, due to their physiological similarities to humans; certain anatomical aspects such as the cerebral gyrencephalic surface of their brains, that allow better examination of the grey-white matter and physiological similarities within the hemoglobin dissociation curve [22]. Additionally, a robust experience using this animal model [23-25] supported its selection. A convenience sample of 12 Ovis Aries wethers weighing $40 \pm 5 \mathrm{k}$ were instrumented with a triple lumen central line (Cook Medical INC. QLD, Australia) and two 16 Fr introducer sheaths in the right internal jugular (RIJ) vein. General anesthesia was given through the central line: ketamine with an initial bolus of $5 \mathrm{mg} / \mathrm{k}$ and maintenance infusion at $0.5-1 \mathrm{mg} / \mathrm{k} / \mathrm{h}$; and infusions of midazolam $(0.5 \mathrm{mg} / \mathrm{k} / \mathrm{h})$, fentanyl $(10 \mathrm{mcg} / \mathrm{k} / \mathrm{h})$, and alfaxalon $(6 \mathrm{mg} / \mathrm{k} / \mathrm{h})$, previously used [26] demonstrating that this anesthetic combination maintains cardiovascular stability without altering cerebral microcirculation [27]. Hydration was ensured with Hartmann's solution, titrated to maintain a central venous pressure (CVP) of 6-10 mmHg. Cardiovascular monitoring included cardiac output and vascular resistances via a Swan-Ganz catheter as previously described [26, 28]. A 5F umbilical vessel catheter (Argyle, Tyco HealthCare, Mansfield, MA, USA), placed in the right femoral artery was used to allow the withdrawal of blood for the referral sample, at a rate of $10 \mathrm{ml} / \mathrm{min}$. Orotracheal intubation used a size $10 \mathrm{~mm}$ endotracheal tube (SIMS Portex, UK). Sheep were ventilated at $12 \mathrm{bpm}$ with tidal volumes of $8 \mathrm{~mL} / \mathrm{kg}$ and $5 \mathrm{cmH}_{2} \mathrm{O}$ of PEEP with an initial $\mathrm{FiO} 2$ of 1.0 . FiO2, and respiratory rate were titrated to maintain a partial pressure of oxygen $\left(\mathrm{PaO}_{2}\right)$ of $>95 \mathrm{mmHg}$ and normocapnia. PEEP levels were maintained at $5 \mathrm{cmH}_{2} \mathrm{O}$ to minimise de-recruitment while known not to effect on cerebral blood flow [26, 29].

Neuro-monitoring used a Lycox $\mathrm{PtiO}_{2}$ probe and an intracranial pressure (ICP) monitor, Oxford Optronics, Ltd., Oxford, UK. Craniectomies were performed prior to injury but dura was not pierced until after the injury was elicited, to avoid the potential vent of intracranial pressure. Craniotomies for the insertion of both probes were performed exactly $15 \mathrm{~mm}$ lateral to the sagittal suture and anterior to the coronal suture $[25,30]$. Probes were introduced at $35 \mathrm{~mm}$ and $15 \mathrm{~mm}$ from the skull respectively with the end of the tip located at the white matter as previously performed [31].

To avoid red blood cell storage into the sheep's spleen and maintaining stable hemoglobin throughout the study [32, 33], the ligation of the splenic artery was performed as reproduced from previous studies [26, 31].

The monitoring and preparation phase was completed with an intracardiac echocardiography (ICE) guided insertion of a transseptal catheter into the left cardiac atrium (LA). Echocardiography images were obtained using an Acuson Sequoia C512 scanner (Siemens, California). Transeptal puncture and insertion of a pigtail catheter into the LA followed previously described methods [34].

\section{Trauma model}

Under non-recovered anesthesia and with the animal's head in a sphinx's position, a blunt injury was applied over the left temporal bone using a non-penetrating stunner (model MKL, Karl Schermer, Ettlingen Germany) with the intention to induce a severe 
head injury. This model followed the same methodology than in a previous study [26], leading to a final combination of contusion and acceleration injury [35].

\section{Normovolemic anemia model}

The targeted hemoglobin goal was defined at each subject, depending upon their post-spleen ligation hemoglobin levels. The aim was to achieve a $30 \%$ reduction on the animal's hemoglobin, from their baseline. Baseline hemoglobin was defined as the post-spleen ligation hemoglobin once stability of the intravascular hemoglobin was achieved. The rationale behind the achievement and maintenance of such reduction was to reproduce the same proportional decay in hemoglobin as the "anemia threshold" previously described in the TRICC Trial [10]. Acute normovolemic anemia was achieved by sequential blood extractions from the indwelling arterial catheter, with simultaneous isovolemic saline infusions to ensure normovolemia. Extracted blood was stored into a Leukotrap ${ }^{\circledR}$ RC System (Baintree MA, 02184, USA) and maintained at room temperature. The cardiovascular response to anemia was monitored using cardiac index, blood pressure and systemic vascular resistances to ensure that the shift on intravascular volume was not leading to intravascular depletion. Regular arterial blood gas sampling was used to ensure stable and normal lactate levels; cardiac monitoring was recorded continuously and in real time while blood gases were performed every hour.

\section{Self-transfusion model}

Once the targeted anemia had been achieved and maintained for over $2 \mathrm{~h}$ allowing physiological and metabolic responses to establish, animals were self-transfused. Labelled and identified Leukotrap ${ }^{\bullet}$ bags were selected for each subject and connected to their central lines. Blood was transfused over $30 \mathrm{~min}$ while cardiovascular monitoring was continuously recording. An arterial blood gas was performed before, during and after the transfusion to avoid over-transfusion, specifically if hemoconcentration was present, in which case, $250 \mathrm{ml}$ volume crystalloid aliquots was administered to achieve the level of hemoglobin closest to each baseline value.

\section{Protocol for microspheres injection}

Interventions were distributed along different time epochs, as shown in Table 1. Along these time points (T0-T4) an injection of color-coded microspheres (E-Z TRAC; Interactive Medical Technology, Los Angeles, CA, USA) was done through the LA pigtail catheter as previously performed [26, 31, 36]. Randomly assigned color at each time-point and subject minimised selection biases and allowed the tracking of RMBF at specific anatomical regions for each time-point and subject. Five different colors (purple low, purple high, pink high, yellow high, and coral low) were recommended by the manufacturer www.microspheres.net to facilitate cytometric count. Each injection included a homogeneous mixture of one color-microspheres of a density equal to 5 million spheres in a $0.8 \mathrm{~mL}$. This microsphere density has been seen not to cause microvascular occlusion [37].

Microspheres were injected $30 \mathrm{~s}$ after the initiation of the withdrawal pump. The withdrawal pump was connected to the arterial catheter with the intention to withdraw blood at a rate of $10 \mathrm{~mL} / \mathrm{min}$ to obtain the reference blood sample required for the 
Table 1 Final study (4 and 5 of 5 studies) interventions distributed along different time points

\begin{tabular}{ll}
\hline Time & Series of chronological interventions \\
\hline T0 & Central lines, intubation, and ventilation set up. \\
& Infusions and intracardiac catheterization. \\
& Arterial catheter and withdrawal pump set up. \\
& Spleen ligation. \\
& Baseline injection of color-coded microspheres \\
& Burr-holes completion \\
T1 & Head injury model \\
& PTio2 and ICP proves insertion \\
T2 & First injection of color-coded microspheres \\
T3 & Anemia model \\
& Second injection of color-coded microspheres \\
T4 & Third injection of color-coded microspheres \\
& Self-transfusion model \\
& Fourth injection of color-coded microspheres \\
& Euthanasia and organ retrieval \\
\hline
\end{tabular}

Italics refer to the phases through which each experiment goes through

calculation of regional tissue RMBF. 2 min after commencement of the withdrawal pump the reference blood sample collection was completed, and the inline catheter was flushed with Tween 80 reagent to recover all the microspheres that could be entrapped in the line [38].

\section{Euthanasia and post-mortem tissue manipulation}

At the end of the study time and approximately 30 min after T4 allowing a systemic distribution of microspheres, sheep were euthanized under non-recovered anesthesia with a bolus injection of $0.5 \mathrm{~mL} / \mathrm{k}$ of sodium pentobarbitone. After confirmation of death (defined as asystole), the brain was extracted, weighed, and fixed with 10\% formalin for 3 weeks.

\section{Brain harvesting technique}

Brain harvesting required the use of a round reciprocating saw to facilitate skull sections of approximately $5 \mathrm{~cm}$. These sections were performed from the frontal region to the posterior fossa. These bone sections were removed whilst simultaneous dissection of the dura avoided parenchymal tearing. Once the brain was fully exposed, the olfactory bulbs, optic chiasm, tentorium, and cranial nerves were progressively sectioned as the brain was lifted from the base of the skull. This approach achieved a controlled dissection avoiding injury to the brain tissue and ensuring a cautious brain removal as previously demonstrated [27]. The brains were weighed prior to insertion into a formalin bath for an immersion fixation during a minimum of 3 weeks.

\section{Tissue sampling model}

After 3 weeks minimum of immersion fixation, the brains were macroscopically inspected to assess for cortical impacts, hemorrhages or the presence of contra-coup injury. Following external inspection, each brain was sectioned creating $5 \mathrm{~mm}$ 
antero-posterior slices. Each slice was macroscopically inspected to identify regions of maximal contusion. Cone samples were extracted from pre-defined anatomical regions (Table 2) as previously published [26, 31]. Adjacent tissue blocks were assigned for both cytometric and histological analysis, to superimpose histology with cerebral blood flow data and allow a flow/tissue cerebral mapping.

Samples from the skin, kidney, heart, and spleen were extracted from each sheep to demonstrate systemic distribution of microspheres as well as to confirm the presence of splenic infarct related to a successful spleen ligation respectively.

\section{Quantification of microvascular blood flow}

Cytometric analysis directly quantified the amount of microspheres of each particular color at each specific region of interest [39]. RMBF was calculated as a mathematical derivation from the microsphere concentration that was injected into the heart at each injection time and the amount of each color microspheres found at each "reference sample of blood." The reference sample of the blood is the arterial blood sample withdrawn at a known rate over a fixed period of time. RMBF represents the proportion of microspheres trapped in the targeted tissue in relation to the total quantity of spheres per milliliter of blood per minute on the reference sample, it uses the following equation:

$\operatorname{RMBF}(\mathrm{mL} / \mathrm{min} / \mathrm{g})=($ total tissue spheres $) /[$ (tissue weight, $\mathrm{g}) \times($ reference spheres $/$ $\mathrm{mL} / \mathrm{min})]$ [40].

Cytometric analysis was performed at the Interactive Medical Technology (IMT), Los Angeles, CA, USA, www.microspheres.net.

\section{Immunohistochemistry processing}

Immunohistochemistry analysis was performed at the neuro-pathology laboratory, Royal Brisbane and Women's' Hospital, QLD, Australia. Immunohistochemistry used a Leica Novolink Polymer Detection Systems Kit (Leica Microsystems Pty Ltd., North Ryde, 2113 Australia) as per manufacturer's instructions, www.leica-microsystems.com. Sections had paraffin removed through a series of xylene immersions and re-hydrations. Antigen retrieval was carried out using Leica BOND ER1 solution. Sections were incubated with a protein block. The primary antiserum made up in Leica BOND Antibody Diluent was applied to the sections.

Immunohistochemistry and hematoxillin and eosin scoring, and interpretation Immunohistochemistry analysis using APP antibodies staining was applied to all targeted areas of interest. APP antibody staining was used to identify areas of tissue with

Table 2 Tissue sampling labelling

\begin{tabular}{ll}
\hline Anatomical regions & Anatomical location \\
\hline AL & Core of contusion, left side \\
BL & Ischemic penumbra, left side \\
AR & Mirror region to core of contusion, on the right \\
BR & Mirror region to ischemic penumbra, on the right \\
C & Thalamus ipsilateral to injury \\
D & Medulla \\
\hline
\end{tabular}


high density of APP staining, specifically at regions of interest. APP expression is considered to be a very early marker of neuronal damage [41] and therefore suitable as an early histopathological marker, interesting in the setting of this 4-h study. A grading system of the density of APP staining [27] was used. APP score was structured into three qualitative categories dependent upon the severity of injury seen: mild, a focal contusion with APP labelling limited to the site of injury or focal APP labelling; moderate, a pattern of APP staining greater than one hemisphere, greater than half a hemisphere or less than half a hemisphere; severe, characterised for the presence of diffuse staining and sub-classified as either diffuse vascular injury, diffuse axonal injury with macroscopic hemorrhage, diffuse axonal injury with microscopic hemorrhage/tissue tears or diffuse axonal injury only [27]. Each animal had samples for both cytometric count of RMBF and immunohistochemistry at each anatomical region of interest with the intention to superimpose flow data with histopathology data at each area of interest and at each time point before and after severe head injury.

\section{Statistical analysis}

RMBF raw data for each sheep was plotted over time and then time averaged for the study cohort. The ratio of RMBF from 1 to $4 \mathrm{~h}$ after injury (T1-T4) compared to baseline (time zero-T0) was plotted. A ratio of one indicated no RMBF changes pre-post injury. RMBF values below the ratio of one indicted that RMBF was reduced over time from baseline; RMBF values above the ratio indicated an increase in RMBF over time from baseline. To test for statistical differences, we used a mixed-effect regression model of the ratios from times $\mathrm{T} 1$ to $\mathrm{T} 4$ with a random intercept for each sheep to control for repeated responses from the same sheep. We fitted an independent effect at each time ( $\mathrm{T} 1$ to $\mathrm{T} 4$ ) as we were uncertain of how the change in RMBF over time would be. We also examined a simpler model where the RMBF ratio was the same at times $\mathrm{T} 1$ to $\mathrm{T} 4$.

All the plots and regression models were run separately for each area studied (AR, $\mathrm{BR}, \mathrm{AL}, \mathrm{BL}, \mathrm{C}$ and $\mathrm{D})$. We used the R software version 3.1.2 for all analyses.

Statistical analyses also assessed the differences between RMBF after head injury during the anemia phase (T2 and T3) and after blood transfusion (T4).

\section{Results}

A convenience sample of 12 subjects weighing $40-45 \mathrm{k}$ was used in this study. All subjects except one (subject number 7) remained cardio-vascularly stable throughout the study time (Table 3). Subject number 7 became hypotensive requiring metaraminol infusion to support his cerebral perfusion pressure (CPP); such profound vasoplegia immediately after trauma was suggestive of cerebral herniation. This observation is corroborated by the cytometric quantification of RMBF that demonstrated undetectable RMBF at all time-points and regions during the study (Fig. 1). Systemic variables affecting CPP and reflecting peripheral oxygen extraction, such as cardiac output (CO) and central venous oxygen saturation (SVc02) respectively, were maintained homogeneously within each subject for the entire study period (Table 3). A specific attention was given to normocapnia with the goal of avoiding hypocapnia due to its known effects over cerebral blood vessel calibre, leading to vasoconstriction with the potential contributory 
Table 3 Mean arterial blood pressure $(\mathrm{mmHg})$, intracranial pressure and cerebral perfusion pressure $(\mathrm{mmHg})$ mean values in all subjects at each time point

\begin{tabular}{|c|c|c|c|c|c|}
\hline $\mathrm{MAP} / \mathrm{ICP} / \mathrm{CPP}(\mathrm{mmHg})$ & T0 pre-injury & $\mathrm{T1}$ & $\mathrm{T} 2$ & T3 & T4 \\
\hline SV02 (\%)/CCO (L/min) & MAP & & & & \\
\hline \multirow[t]{2}{*}{ Subject 1} & 97 & $114 / 43 / 71$ & $76 / \mathbf{2 3} / 53$ & $74 / \mathbf{2 4} / 50$ & $79 / \mathbf{2 9} / 50$ \\
\hline & $72 / 3.1$ & $75 / 3.0$ & $74 / 2.6$ & $77 / 3.6$ & $84 / 4.7$ \\
\hline \multirow[t]{2}{*}{ Subject 2} & 98 & $74 / \mathbf{2 3} / 51$ & $91 / \mathbf{3 0} / 61$ & $79 / \mathbf{2 9} / 50$ & $92 / \mathbf{4 2} / 50$ \\
\hline & $\mathrm{NA} / 2.5$ & NA/2.4 & NA/2.5 & NA/2.5 & NA/3.0 \\
\hline \multirow[t]{2}{*}{ Subject 3} & 110 & 100/NA/NA & 90/NA/NA & 130/NA/NA & 125/NA/NA \\
\hline & $75 / 3.4$ & $79 / 3.4$ & $66 / 2.9$ & $69 / 3.1$ & $77 / 4.1$ \\
\hline \multirow[t]{2}{*}{ Subject 4} & 117 & $106 / 23 / 83$ & $137 / 23 / 114$ & 130/23/107 & $135 / \mathbf{2 5} / 110$ \\
\hline & $70 / 4.6$ & $70 / 4.7$ & $71 / 3.8$ & $72 / 3.9$ & $74 / 5.7$ \\
\hline \multirow[t]{2}{*}{ Subject 5} & 114 & $97 / 23 / 74$ & $72 / \mathbf{2 1 / 5 1}$ & $79 / \mathbf{2 5} / 54$ & $94 / 27 / 67$ \\
\hline & $85 / 4.0$ & $79 / 3.6$ & $78 / 3.7$ & $80 / 3.7$ & $86 / 5.9$ \\
\hline \multirow[t]{2}{*}{ Subject 6} & 90 & $99 / 12 / 87$ & 100/13/87 & $92 / 13 / 79$ & $81 / 10 / 71$ \\
\hline & $77 / 3.2$ & $73 / 3.2$ & $66 / 2.3$ & $61 / 2.3$ & $86 / 5.7$ \\
\hline \multirow[t]{2}{*}{ Subject 7} & 90 & $70 / \mathbf{2 2} / 48^{\mathrm{a}}$ & $70 / 35 / 35^{\mathrm{a}}$ & $80 / 46 / 34^{a}$ & $90 / 67 / 23^{a}$ \\
\hline & $92 / 4.6$ & $98 / 4.1$ & $98 / 4.7$ & $97 / 3.3$ & $98 / 3.9$ \\
\hline \multirow[t]{2}{*}{ Subject 8} & 110 & $80 / 7 / 73$ & 79/6/73 & $90 / 4 / 86$ & $95 / 4 / 91$ \\
\hline & $83 / 4.8$ & $87 / 4.3$ & $87 / 5.1$ & $86 / 4.5$ & $87 / 6.2$ \\
\hline \multirow[t]{2}{*}{ Subject 9} & 90 & $90 / 8 / 82$ & $94 / 6 / 88$ & $95 / 5 / 90$ & $103 / 4 / 99$ \\
\hline & $54 / 2.9$ & $51 / 3.1$ & $53 / 3.2$ & $66 / 3.7$ & $69 / 5.5$ \\
\hline \multirow[t]{2}{*}{ Subject 10} & 72 & $94 / 6 / 88$ & 97/5/92 & $77 / 4 / 73$ & 109/4/105 \\
\hline & $\mathrm{NA} / 2.7$ & NA/3.0 & NA/3.0 & NA/4.1 & NA/5.6 \\
\hline \multirow[t]{2}{*}{ Subject 11} & 130 & 133/2/131 & $124 / 5 / 119$ & 108/3/105 & $128 / 3 / 125$ \\
\hline & $74 / 3.3$ & $76 / 3.6$ & 70/3.0 & $78 / 4.3$ & $80 / 5.4$ \\
\hline \multirow[t]{2}{*}{ Subject 12} & 107 & 125/26/99 & $118 / 21 / 97$ & $90 / 19 / 71$ & $114 / 13 / 101$ \\
\hline & $71 / 3.4$ & $71 / 3.2$ & $72 / 3.1$ & $71 / 3.2$ & $72 / 4.0$ \\
\hline
\end{tabular}

MAP mean arterial pressure, ICP intracranial pressure, CPP cerebral perfusion pressure, calculated as per MAP-ICP. T0 shows only values of MAP as ICP probe was inserted after injury (T1). Patients with high ICP indicated in bold, ${ }^{a}=$ CPP below $50 \mathrm{mmHg}$ requiring metaraminol infusion. Venous saturation of oxygen (Sv02) expressed in \%. Continuous cardiac output $(\mathrm{CCO})$ expressed in liters per minute $(\mathrm{L} / \mathrm{min})$. Bold values indicate ICP higher then normal. Italic values indicate the increase in Cardiac Output after transfusion

effect of hypoperfusion. Metabolic variables reflecting oxygen delivery to tissues as well as impacting on microcirculation rheology, such as the hemoglobin level is represented in Table 4 (Additional file 1). During T2 and T3, a 30\% reduction in each subject's baseline hemoglobin was achieved as per protocol. Subsequent self-transfusion was completed on T4, showing the increment on hemoglobin in 8 of the 12 subjects studied. $\mathrm{PtiO}_{2}$ was recorded in every subject from $\mathrm{T} 1$, as $\mathrm{PtiO}_{2}$ probes were inserted after craniectomies were formalised (Table 5). $\mathrm{PtiO}_{2}$ at $\mathrm{T} 1$ was seen to very critically low in some subjects; this is a common observation following the insertion of the probe. In seven of 12 subjects, $\mathrm{PtiO}_{2}$ values were persistently below the described ischemic threshold [4-7] throughout the trauma and anaemia phase. In all the subjects except for two (subject numbers 2 and 7), $\mathrm{PtiO}_{2}$ values significantly incremented and recovered after self-transfusion. At time $\mathrm{T} 4$, the $\mathrm{PtiO}_{2}$ was 9.6 times higher on average, $95 \%$ CI 5.6 to 13.6 ( $p$ value $<0.001$ ), this observation is consistent with previous studies. 

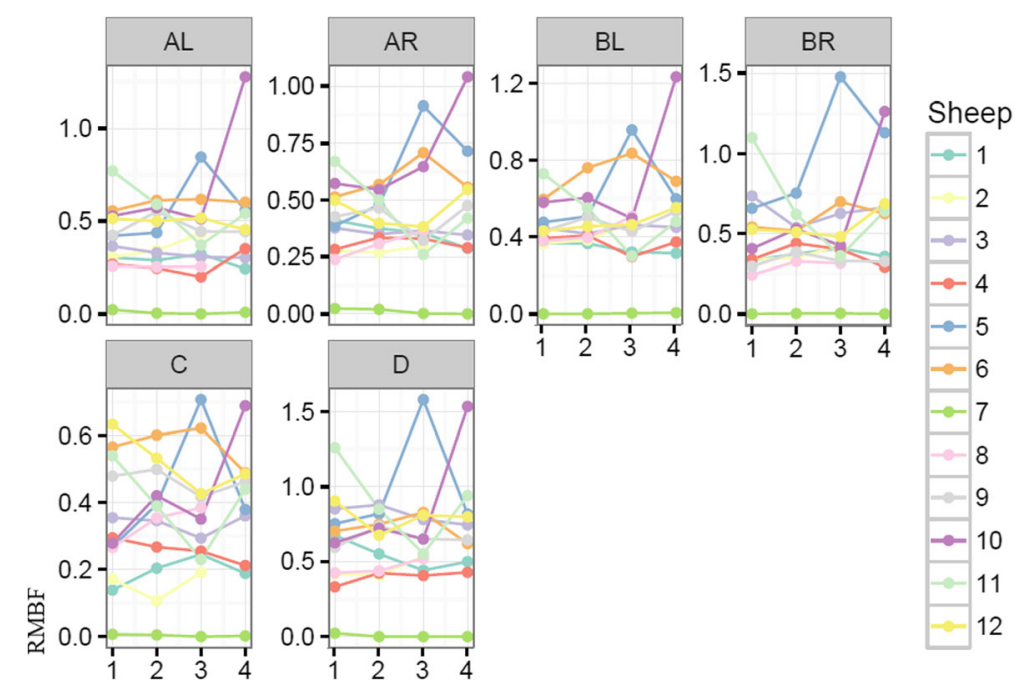

Fig. 1 Regional microcirculation blood flow (RMBF) ratio from baseline for all subjects at each anatomical region of interest over time. Time-points (T1-T4) on the $x$ axis; RMBF per $1 \mathrm{~g}$ tissue on the $y$ axis

\section{RMBF analysis}

Each sheep had a baseline RMBF measure prior to injury (time zero-T0) and subsequent hourly RMBF measures every hour for $4 \mathrm{~h}$. RMBF values for each subject and tissue region over the entire study time are represented in Fig. 1 with their means represented in Fig. 2; showing that in all subjects, RMBF from baseline to T3 was reduced to ischemic thresholds $(20-30 \mathrm{ml} / 100 \mathrm{~g} / \mathrm{min})$ with a considerable increase beyond normal RMBF ranges after transfusion. The ratio of RMBF from T1 (post injury) to T2-T3 (post anaemia) and T4 (post-transfusion) compared to baseline (time zero-T0) was also plotted. A ratio of one indicates absence of changes in RMBF throughout interventions when compared with baseline; RMBF values below the ratio of one represents a

Table 4 Hemoglobin levels $(\mathrm{g} / \mathrm{dL})$ for all subjects over time

\begin{tabular}{llllll}
\hline Subjects & Times & & & & \\
\cline { 2 - 6 } & T0 & T1 & T2 & T3 & T4 \\
\hline Sheep 1 & 10.6 & 10.0 & 7.5 & 7.6 & $\mathbf{9 . 1}$ \\
Sheep 2 & 8.0 & 8.2 & 6.4 & 6.4 & $\mathbf{7 . 6}$ \\
Sheep 3 & 7.0 & 7.0 & 5.6 & 5.1 & $\mathbf{6 . 9}$ \\
Sheep 4 & 8.4 & 8.2 & 6.8 & 6.7 & $\mathbf{7 . 9}$ \\
Sheep 5 & 7.7 & 7.5 & 5.2 & 5.5 & $\mathbf{7 . 0}$ \\
Sheep 6 & 8.6 & 6.5 & 6.7 & 6.4 & $\mathbf{8 . 0}$ \\
Sheep 7 & 9.2 & 9.0 & 6.6 & 6.5 & $\mathbf{8 . 0}$ \\
Sheep 8 & 8.5 & 9.3 & 6.5 & 6.3 & $\mathbf{7 . 9}$ \\
Sheep 9 & 9.8 & 8.0 & 6.6 & 6.5 & $\mathbf{7 . 0}$ \\
Sheep 10 & 8.7 & 8.9 & 6.6 & 6.7 & 7.7 \\
Sheep 11 & 9.0 & 8.1 & 6.4 & 6.9 & 7.9 \\
Sheep 12 & 8.2 & & 6.2 & 7.0 \\
\hline
\end{tabular}

Hemoglobin levels at baseline (T0) and after trauma (T1) were reduced by $30 \%$ on each subject and maintained during the anaemia phase (T2 and T3) followed by self-transfusion (T4). Hemoglobin for the subjects that experienced an increase in their $\mathrm{Hbl}$ after transfusion are indicated in bold 
Table 5 Partial pressure of tissue oxygenation $\left(\mathrm{PtiO}_{2}\right)$ expressed in $\mathrm{mmHg}$ for all subjects over time

\begin{tabular}{llllll}
\hline PtiO $_{2}(\mathrm{mmHg})$ & $\mathrm{T} 0$ & $\mathrm{~T} 1$ & $\mathrm{~T} 2$ & $\mathrm{~T}$ & $\mathrm{~T}$ \\
\hline Sheep 1 & $\mathrm{NA}$ & 6.3 & 2.8 & 2.5 & $\mathbf{1 1 . 4}$ \\
Sheep 2 & NA & 0.8 & 1.6 & 1.0 & 1.67 \\
Sheep 3 & NA & 2.7 & 1.6 & 0.7 & $\mathbf{1 0 . 2}$ \\
Sheep 4 & NA & 14.6 & 17.9 & 24.1 & $\mathbf{3 4 . 4}$ \\
Sheep 5 & NA & 0.1 & 1.4 & 1.9 & $\mathbf{1 0 . 6}$ \\
Sheep 6 & NA & 3.2 & 3.1 & 2.7 & $\mathbf{1 0 . 6}$ \\
Sheep 7 & NA & 20 & 35 & 23 & 17 \\
Sheep 8 & NA & 4.2 & 6.4 & 15 & $\mathbf{3 4 . 3}$ \\
Sheep 9 & NA & 15.5 & 26.6 & 28.9 & $\mathbf{3 4 . 8}$ \\
Sheep 10 & NA & 14.4 & 6.4 & 9.7 & $\mathbf{2 2 . 9}$ \\
Sheep 11 & NA & 42 & 31.4 & 27.5 & $\mathbf{3 7 . 7}$ \\
Sheep 12 & NA & 49.9 & 34.5 & 40 & $\mathbf{6 3}$ \\
\hline
\end{tabular}

T0 represents pre-injury phase in which craniectomies are not formalized. $\mathrm{PtiO}_{2}$ probes were inserted after trauma, recording $\mathrm{PtiO}_{2}$ values only from $\mathrm{T} 1$

$\mathrm{T} 2$ and $\mathrm{T} 3$ italic values represent $\mathrm{PtiO}_{2}$ values below the theoretical critical ischemic threshold

$\mathrm{PtiO}_{2}$ at $\mathrm{T} 4$ bold values were significantly increased when compared with pre-transfusion times

reduction in RMBF over time from baseline; RMBF values above such ratio represents an increase in RMBF over time from baseline Fig. 3. RMBF mean ratios from baseline of all subjects, anatomical region, and time are represented in Fig. 4. These two previous figures show that while most subjects were distributed along the ratio of one over all time-points; all their RMBF means at $\mathrm{AL}, \mathrm{BL}, \mathrm{AR}$, and $\mathrm{BR}$ were reduced from baseline to post-injury and anemia phase (T1 to T3) but increased from baseline during transfusion phase (T4). Conversely, the anatomical regions showing a mean RMBF increase from baseline were the ipsilateral thalamus (C) and medulla (D). RMBF means per region and time with the 95\% confidence intervals represented by the vertical lines are shown in Fig. 5; statistical significance is represented by those confidence intervals that do not cross the horizontal reference line of 1 .

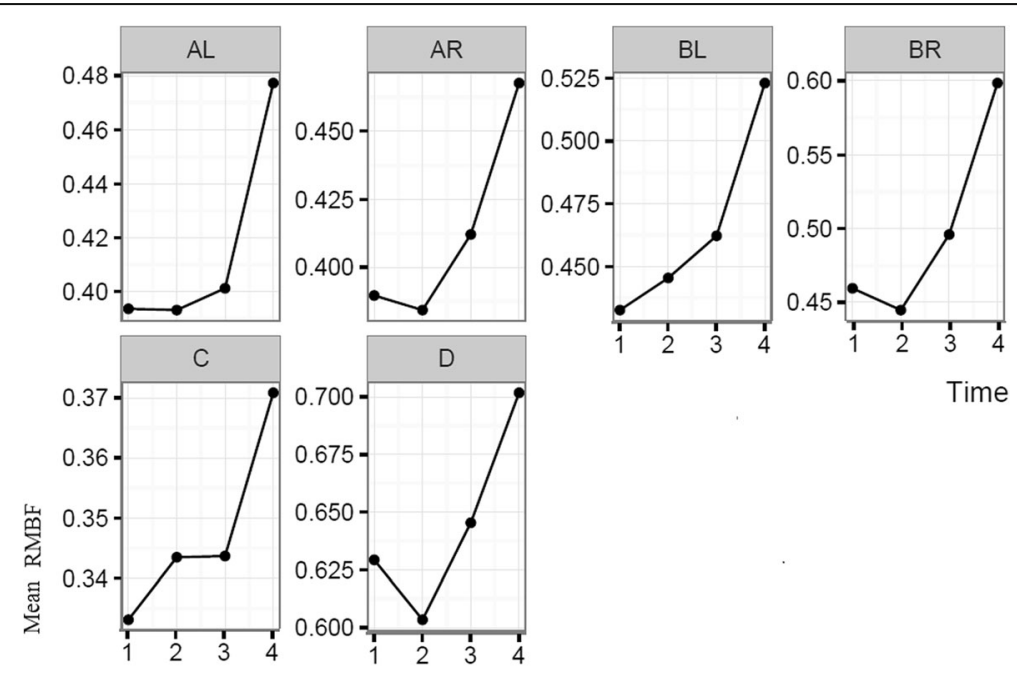

Fig. 2 Mean regional microcirculation blood flow (RMBF) from baseline for all subjects at each anatomical region of interest, over time. Time-points (T1-T4) on the $x$ axis; mean RMBF on the $y$ axis 

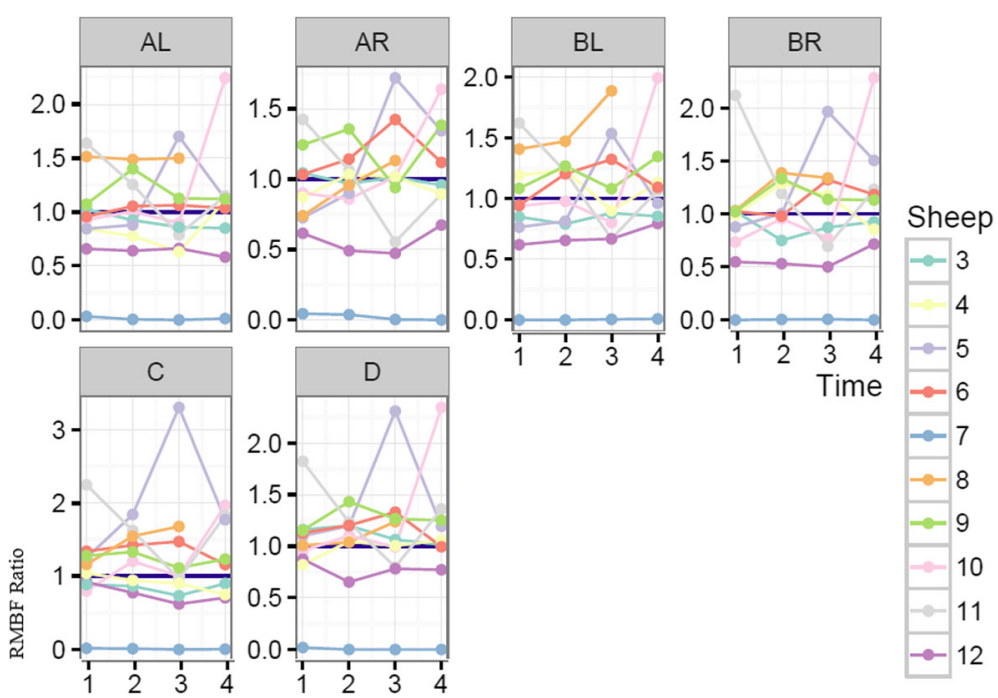

Fig. 3 Regional microcirculation blood flow (RMBF) ratio from baseline (y axis), per subject and anatomical region of interest, over time ( $x$ axis)

\section{APP scoring}

Results for APP scoring are represented in Table 6. Mild APP staining pattern was seen predominantly in all anatomical regions with moderate and severe APP staining pattern also present and distributed homogeneously over all anatomical regions, except for ipsilateral thalamus showing a predominant "moderate" qualitative score for axonal injury.

\section{Discussion}

The most important finding in this study is the consistent reduction of the mean RMBF from baseline (RMBF pre-injury or T0) and after injury, in all subjects. In addition, the RMBF nadir was found to be located at the core of the contusion, and ongoing RMBF reductions were identified at the ischemic penumbra and at contralateral sites to the

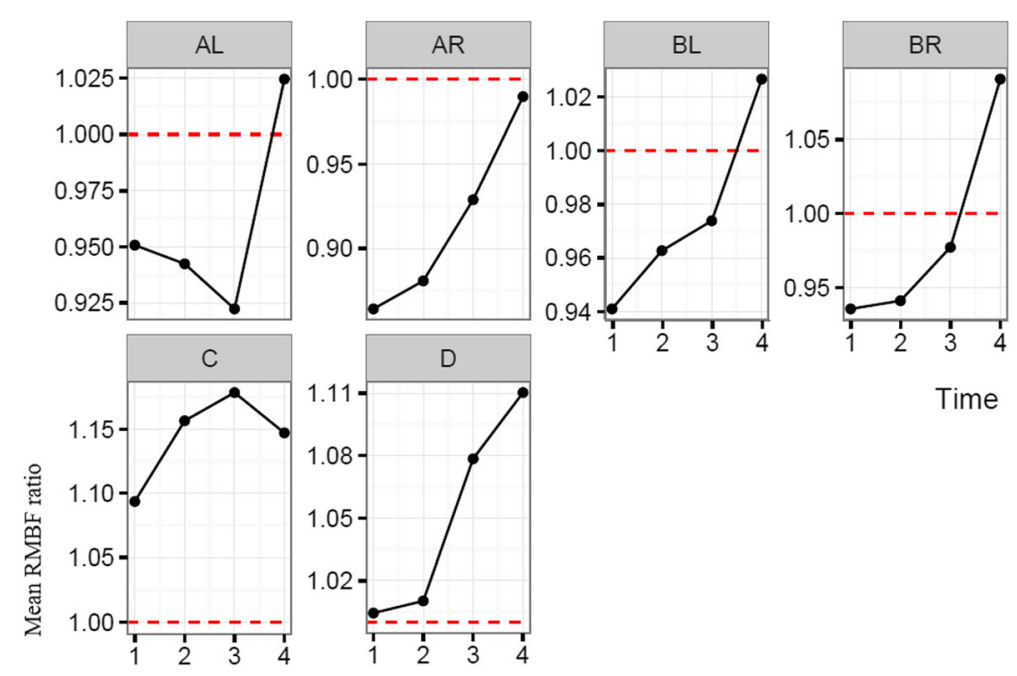

Fig. 4 Mean regional microcirculation blood flow (RMBF) ratio from baseline (y axis), for all subjects at each anatomical region of interest, over time ( $x$ axis) 


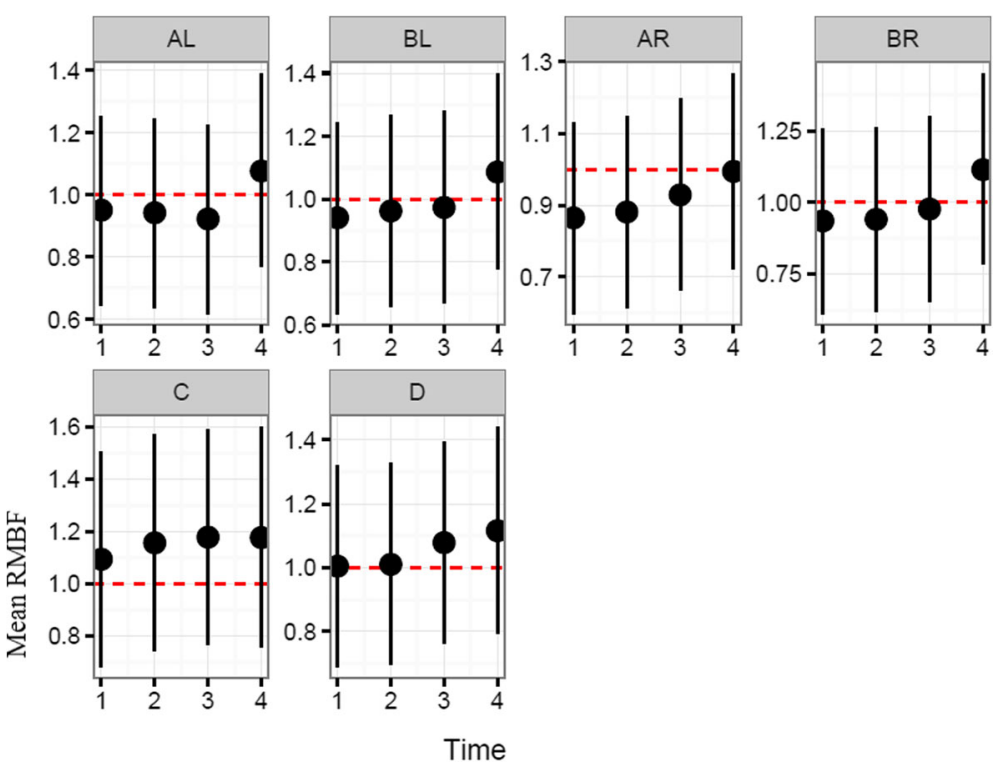

Fig. 5 Mean regional microcirculation blood flow (RMBF) ratio from baseline (y axis) and standard deviation, for all subjects at each anatomical region of interest over time ( $x$ axis)

contusion (Fig. 4). This is consistent with the a priori expectations regarding cerebral microcirculation after severe head injury although this reduction in RMBF was not seen to be statistically significant (Fig. 5). It is likely that the lack of statistical significance relates to the limited cohort of subjects used in this study; however, although statistical significance adds methodological robustness, experimental studies focus their relevance in their design and plausibility of findings, both of which are demonstrated in this study. These findings contrast with the increase in RMBF from baseline, at the ipsilateral thalamus (C region) and medulla (D region). A possible explanation to the sustained increase in RMBF at these two anatomical regions may be related to the physiological distribution of cerebral blood flow and microcirculation in these areas. Blood supply at the thalamus, derives from multiple arteries (posterior communicating artery, the posterior cerebral artery, the choroidal arteries and in some individuals the Percheron artery). Likewise, blood supply to the medulla, also derives from multiple arteries (the anterior spinal artery, the posterior spinal artery, and direct branches of the vertebral artery). These anatomical peculiarities may be a contributing factor to ensuring blood supply to these two pivotal and confluent anatomical regions of the brain.

Table 6 Amyloid precursor protein (APP) staining qualitative scores by tissue region after a severe head injury model

\begin{tabular}{llll}
\hline Anatomical regions & \multicolumn{3}{l}{ Number of subjects categorised on each qualitative APP score } \\
\cline { 2 - 4 } & Mild & Moderate & Severe \\
\hline AL & 11 & 1 & 0 \\
AR & 9 & 2 & 1 \\
BL & 8 & 3 & 1 \\
BR & 8 & 3 & 1 \\
C & 4 & 7 & 1 \\
D & 10 & 2 & 0 \\
\hline
\end{tabular}


The second most relevant finding in this study is the increase of the mean RMBF at all anatomical regions of interest, after the transfusion phase (T4). It may be that the relative increase in subject's $\mathrm{CO}$ could have led to an improvement in microcirculation and subsequent RMBF. However, these subjects' CPP did not increase as a response to self-transfusion, implying that there was either a compensation through changes on vascular resistances or a variation in cerebral hemodynamics beyond the limits of perfusion pressure.

The third relevant finding was that when RMBF was analysed by ICP groups, those subjects with high ICP (SN 1, SN 2, SN 4, SN 5, and SN 7) had persistently lower RMBF levels than subjects with normal ICP. Furthermore, amongst subjects with high ICP, RMBF was below the ischemic threshold at all-time points and throughout several anatomical regions. Such finding is consistent with patho-physiologic expectations in the context of severe head injury, increasing the internal validity and reliability of our findings.

To elucidate the impact of high ICP on RMBF over time after injury, we analysed the mean RMBF ratio from baseline in two separated cohorts: those subjects with normal ICP and those with raised ICP (Fig. 6) and found that

- Subjects with normal ICP values (defined by an ICP $<20 \mathrm{mmHg}$ ) had an increase in their mean RMBF from baseline after trauma (their mean RMBF was above the ratio of 1) as opposed to subjects with raised ICP, who had a reduced mean RMBF from baseline after trauma (their mean RMBF was below the ratio of 1 ).

- Subjects with normal ICP showed their lowest mean RMBF at T3 (just prior to transfusion and after $2 \mathrm{~h}$ from trauma) and an increase in their mean RMBF at T4 (after self-transfusion) as opposed to subjects with raised ICP.

The time-dependent reduction of cerebral RMBF in all regions from baseline, amongst subjects with raised ICP is a relevant finding as it may indicate that even in

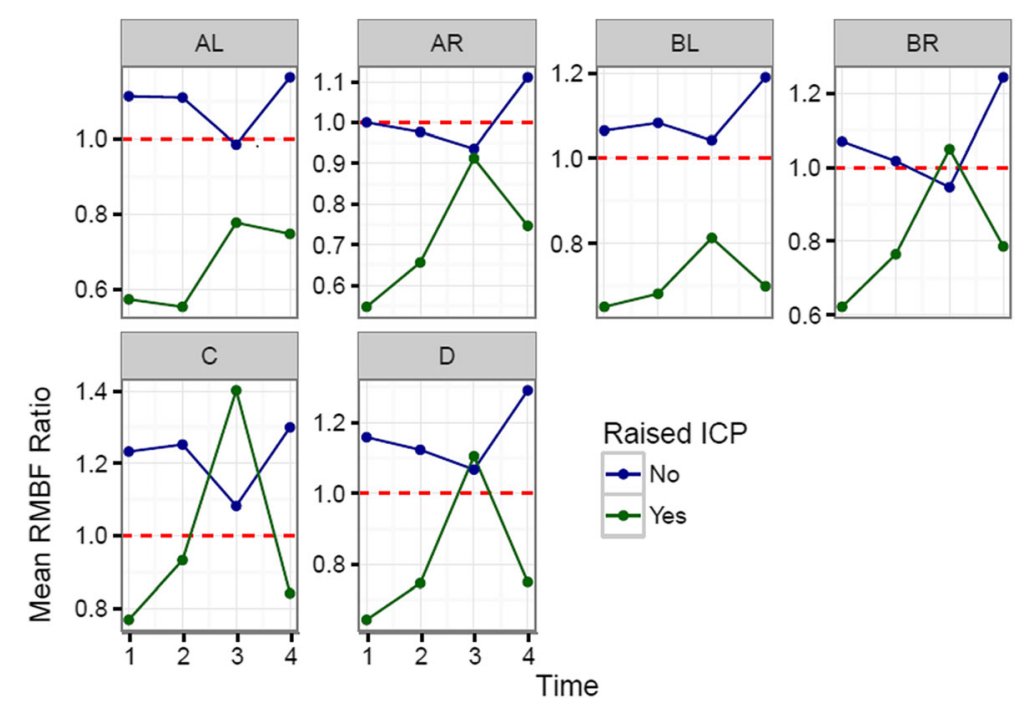

Fig. 6 Mean regional microcirculation blood flow (RMBF) ratio from baseline (y axis), for all subjects at each anatomical region of interest, over time ( $x$ axis), comparing two cohorts of subjects (high ICP versus normal ICP) 
situations where severe tissue disruption is not derived from the primary injury leading to a mild degree of APP staining, there is still the potential for the development of cerebral infarcts.

The fourth relevant finding was that $\mathrm{PtiO}_{2}$ values declined during the anemic phase and had a statistically significant increase after autologous transfusion, in all subjects except for SN 2 and 7, a finding consistent with previous studies [21]. It may be that despite the global reduction in RMBF from baseline, the maintenance of RMBF above ischemic-thresholds may be sufficient to preserve tissue oxygenation; this emphasizes the need to preserve cerebral perfusion even from early hours after trauma.

In addition, histopathological analysis using APP staining (Table 6) showed a mild degree of APP staining not expected from the severity of the injury. However, moderate and severe APP staining scores were also found along all regions including the contralateral hemisphere, demonstrating that axonal injury is globally manifested. Indeed, the presence of axonal retraction balls (ARB) corroborates with the severity of injury, as ARB are considered the hallmark of DAI (Fig. 7). This study compares for the first time the interrelation between tissue damage and microcirculation in experimental models at specific anatomical regions. It is the first time that cerebral histopathology and quantification of microcirculation post-trauma are analysed simultaneously; showing how cerebral blood flow and axonal tissue are temporal and spatially affected after trauma.

These observations are clinically relevant, they demonstrate the increasing vulnerability of cerebral microcirculation not just in relation to the severity of the injury but most importantly to the superposition of additional insults such as anemia and increased ICP. In addition, the significant increase in $\mathrm{PtiO}_{2}$ after transfusion reflects a degree of reversibility and brings the question onto its theoretical role in reducing the risk of cerebral infarcts and improving neurological or functional outcomes.

In summary, in the presence of severe head injury and superimposed anemia, cerebral microcirculation is consistently reduced from baseline with a nadir at the level of the core of the contusion and a maximal reduction at the pre-transfusion phase. In such scenario, when raised ICP is added, cerebral microcirculation is reduced further and does not recover with autologous transfusion. Moreover, during anemia, $\mathrm{PtiO}_{2}$ values are reduced from baseline with a statistically significant increase after autologous transfusion.

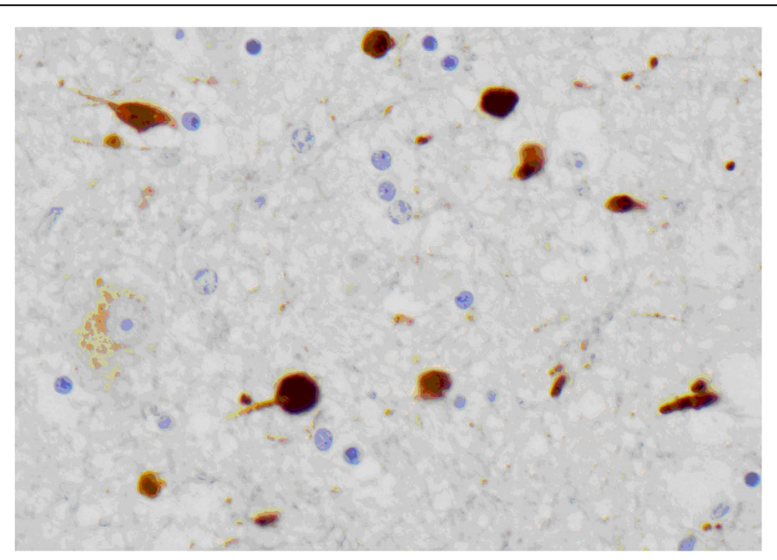

Fig. 7 Axonal retraction balls (ARB) in a subject with severe head injury and diffuse axonal injury 


\title{
Study limitations
}

The biggest limitation of this study relies on the limited capacity to clinically extrapolate conclusions raised from experimental models. However, it is from experimental studies that direct quantification of cerebral microcirculation becomes feasible. Another important limitation relates to the limited length of the time during which the animals were monitored and studied. Hence, although longer monitoring time could have demonstrated a wider range of patho-physiological processes affecting microcirculation, the focus of this study was to capture the early changes in RMBF at specific regions of interest superimposed to the early histopathology changes derived from axonal injury after trauma.

\section{Conclusion}

Cerebral microcirculation after severe head injury is consistently reduced from baseline, at the ipsilateral and contralateral site of the contusion. When normovolemic anemia is superimposed, cerebral microcirculation reaches its nadir with an increase response after autologous transfusion amongst subjects with normal ICP, unlike for subjects with high intracranial pressures. The reduction in cerebral microcirculation is associated with the severe APP staining and the presence of axonal retraction balls, the DAI histological hallmark. Cerebral oxymetry showed a statistically significant increase from ischemic to above-normal values after autologous transfusion.

\section{Additional file}

Additional file 1: Metabolic variables. (DOCX $119 \mathrm{~kb})$

\begin{abstract}
Abbreviations
APP: Amyloid precursor protein; ARB: Axonal retraction balls; CO: Cardiac output; CPP: Cerebral perfusion pressure; CVP: Central venous pressure; ICE: Intracardiac echocardiography; ICP: Intracranial pressure; IMT: Interactive Medical Technology; $\mathrm{PaC0}$ : Partial pressure of arterial $\mathrm{CO}_{2} ; \mathrm{PaO}_{2}$ : Partial pressure of oxygen; $\mathrm{PtiO}_{2}$ : Partial pressure of tissue oxygenation; RMBF: Regional microcirculation blood flow; SVc02: Central venous oxygen saturation
\end{abstract}

\section{Acknowledgements}

The authors thank the Royal Brisbane and Women's' Research Foundation for their funding contribution and the Medical Engineering Research Facility (MERF) team for their logistic support facilitating the surgeries required in this study.

\section{Availability of data materials}

The datasets used and/or analysed during the current study are available from the corresponding author on reasonable request.

\section{Funding}

This study was funded in its entire by the Royal Brisbane and Women's Research Foundation Project Grant January 2016. This funding body was not involved in decisions regarding study design, data analysis, or interpretation.

\section{Authors' contributions}

Primary roles: JB contributed to the study design, data collection, surgical procedures, data interpretation, manuscript drafting, and first edition. KC helped in the histopathological analysis. KD contributed to the laboratory support and manuscript correction. SD carried out the surgical procedures and data collection. DP and CR helped in the intracardiac echography and transseptal catheterization. LG carried out the manuscript preparation. AB helped in the statistical analysis. JP, RB, and JFF contributed to the data interpretation and manuscript correction. All authors read and approved the final manuscript.

Authors' information

Not applicable

\section{Ethics approval}

The study methods were submitted and approved by the Animal Ethics Committee of the Queensland University of Technology and the University of Queensland, Australia. This study was conducted in accordance with the Declaration of Helsinki. 


\title{
Consent for publication
}

Not applicable

\section{Competing interests}

The authors declare that they have no competing interests.

\section{Publisher's Note}

Springer Nature remains neutral with regard to jurisdictional claims in published maps and institutional affiliations.

\begin{abstract}
Author details
${ }^{1}$ Critical Care Research Group, University of Queensland, Brisbane, Queensland, Australia. ${ }^{2}$ Intensive Care Department, Royal Brisbane and Women's Hospital, Butterfield Street, Herston, QLD 4025, Australia. ${ }^{3}$ School of Medicine, University of Queensland, Brisbane, Queensland 4025, Australia. ${ }^{4}$ Griffith University, Parkland Drive, Southport 4215, Australia. ${ }^{5}$ Histopathology Department, Royal Brisbane and Women's Hospital, Herston, QLD 4025, Australia. ${ }^{6}$ Medical Engineering Research Facility, Queensland University of Technology, Stafford Heights, QLD 4053, Australia. ${ }^{7}$ Department of Cardiology, The Prince Charles Hospital, Chermside, QLD 4032, Australia. ${ }^{8}$ Medical School Research Centre, Frome road, Adelaide, SA 5005, Australia. ${ }^{9}$ Institute of Health and Biomedical Innovation and School of Public Health and Social Work, Queensland University of Technology, 60 Musk Avenue, Kelvin Grove, QLD 4059, Australia.

${ }^{10}$ Intensive Care Department, The Prince Charles Hospital, Rode road, Chermside, QLD 4032, Australia.
\end{abstract}

\section{Received: 23 February 2018 Accepted: 18 October 2018}

Published online: 08 November 2018

\section{References}

1. Werner C, Engelhard K (2007) Pathophysiology of traumatic brain injury. Br J Anaesth 99:4-9

2. Menon DK, Coles JP, Gupta AK et al (2004) Diffusion limited oxygen delivery following head injury. Crit Care Med 32: 1384-1390

3. Schwarzmaier SM, Kim SW, Trabold R et al (2010) Temporal profile of thrombogenesis in the cerebral microcirculation after traumatic brain injury in mice. J Neurotrauma 27:121-130

4. Bardt TF, Unterberg AW, Hartl R et al (1998) Monitoring of brain tissue PO2 in traumatic brain injury: effect of cerebral hypoxia on outcome. Acta Neurochir Suppl 71:153-156

5. Narotam PK, Morrison JF, Nathoo N (2009) Brain tissue oxygen monitoring in traumatic brain injury and major trauma: outcome analysis of a brain tissue oxygen-directed therapy. J Neurosurg 111:672-682

6. Stiefel MF, Spiotta A, Gracias VH et al (2005) Reduced mortality rate in patients with severe traumatic brain injury treated with brain tissue oxygen monitoring. J Neurosurg 103:805-811

7. Stocchetti N, Chieregato A, De Marchi M et al (1998) High cerebral perfusion pressure improves low values of local brain tissue $\mathrm{O} 2$ tension (PtiO2) in focal lesions. Acta Neurochir Suppl 71:162-165

8. Kumar P, Thapliyal R, Coshic P et al (2013) Retrospective evaluation of adverse transfusion reactions following blood product transfusion from a tertiary care hospital: a preliminary step towards hemovigilance. Asian J Transfus Sci 7:109-115

9. Murphy GJ, Reeves BC, Rogers CA et al (2007) Increased mortality, postoperative morbidity, and cost after red blood cell transfusion in patients having cardiac surgery. Circulation 116:2544-2552

10. Hebert PC, Wells G, Blajchman MA et al (1999) A multicenter, randomized, controlled clinical trial of transfusion requirements in critical care. Transfusion requirements in critical care investigators, Canadian critical care trials group. N Engl J Med 340:409-417

11. Carson JL, Grossman BJ, Kleinman S et al (2012) Red blood cell transfusion: a clinical practice guideline from the AABB*. Ann Intern Med 157:49-58

12. Carson JL, Hill S, Carless P et al (2002) Transfusion triggers: a systematic review of the literature. Transfus Med Rev 16 187-199

13. McIntyre LA, Fergusson DA, Hutchison JS et al (2006) Effect of a liberal versus restrictive transfusion strategy on mortality in patients with moderate to severe head injury. Neurocrit Care 5:4-9

14. Fortune JB, Feustel PJ, Saifi J et al (1987) Influence of hematocrit on cardiopulmonary function after acute hemorrhage. J Trauma 27:243-249

15. Topley E, Clarke R (1956) The anemia of trauma. Blood 11:357-369

16. Hare GM, Mazer CD, Hutchison JS et al (2007) Severe hemodilutional anemia increases cerebral tissue injury following acute neurotrauma. J Appl Physiol 103:1021-1029

17. Valadka AB, Gopinath SP, Contant CF et al (1998) Relationship of brain tissue PO2 to outcome after severe head injury. Crit Care Med 26:1576-1581

18. Johnston AJ, Gupta AK (2002) Advanced monitoring in the neurology intensive care unit: microdialysis. Curr Opin Crit Care 8:121-127

19. Stiefel MF, Tomita Y, Marmarou A (2005) Secondary ischemia impairing the restoration of ion homeostasis following traumatic brain injury. J Neurosurg 103:707-714

20. Robertson CS, Hannay HJ, Yamal JM et al (2014) Effect of erythropoietin and transfusion threshold on neurologica recovery after traumatic brain injury: a randomized clinical trial. JAMA 312:36-47

21. Bellapart J, Boots R, Fraser J (2012) Physiopathology of anemia and transfusion thresholds in isolated head injury. J Trauma Acute Care Surg 73:997-1005

22. Samaja M, Gattinoni L (1978) Oxygen affinity in the blood of sheep. Respir Physiol 34:385-392

23. Cernak I (2005) Animal models of head trauma. NeuroRx 2:410-422

24. Ommaya AK, Gennarelli TA (1974) Cerebral concussion and traumatic unconsciousness. Correlation of experimental and clinical observations of blunt head injuries. Brain 97:633-654 
25. Van Den Heuvel C, Lewis S, Wong M et al (1998) Diffuse neuronal perikaryon amyloid precursor protein immunoreactivity in a focal head impact model. Acta Neurochir Suppl 71:209-211

26. Bellapart J, Abi-Fares C, Cuthbertson $\mathrm{K}$ et al (2016) Cerebral microcirculation during mild head injury after a contusion and acceleration experimental model in sheep. Brain Inj. 2016;30(13-14):1542-1551. Epub 2016 Aug 26.

27. Bellapart J, Cuthbertson K, Skerman J et al (2014) Colour coded microspheres and histological analysis for cerebral mapping: an experimental model. Journal of Neurology Research 4:7-14

28. Maybauer DM, Maybauer MO, Traber LD et al (2006) Effects of severe smoke inhalation injury and septic shock on global hemodynamics and microvascular blood flow in sheep. Shock 26:489-495

29. Videtta W, Villarejo F, Cohen M et al (2002) Effects of positive end-expiratory pressure on intracranial pressure and cerebral perfusion pressure. Acta Neurochir Suppl 81:93-97

30. Lewis SB, Myburgh JA, Thornton EL et al (1996) Cerebral oxygenation monitoring by near-infrared spectroscopy is not clinically useful in patients with severe closed-head injury: a comparison with jugular venous bulb oximetry. Crit Care Med 24:1334-1338

31. Bellapart J, Cuthbertson K, Dunster K et al (2016) Cerebral Microcirculation during Experimental Normovolaemic Anemia. Front Neurol 7:6

32. Simonova G, Tung JP, Fraser JF et al (2014) A comprehensive ovine model of blood transfusion. Vox Sang 106:153-160

33. Hodgetts VE (1961) The dynamic red cell storage function of the spleen in sheep. III. Relationship to determination of blood volume, total red cell volume, and plasma volume. Aust J Exp Biol Med Sci 39:187-195

34. Bellapart J, Dunster KR, Diab S et al (2013) Intracardiac echocardiography quided transeptal catheter injection of microspheres for assessment of cerebral microcirculation in experimental models. Cardiol Res Pract 2013:595838

35. Finnie JW, Blumbergs PC, Manavis J et al (2000) Evaluation of brain damage resulting from penetrating and nonpenetrating captive bolt stunning using lambs. Aust Vet J 78:775-778

36. Bellapart J, Fraser JF (2009) Transcranial doppler assessment of cerebral autoregulation. Ultrasound Med Biol 35:883-893

37. Traber DL (2000) Animal models: the sheep. Crit Care Med 28:591-592

38. Hodeige D, de Pauw M, Eechaute W et al (1999) On the validity of blood flow measurement using colored microspheres. Am J Phys 276:H1150-H1158

39. Prinzen FW, Bassingthwaighte JB (2000) Blood flow distributions by microsphere deposition methods. Cardiovasc Res 45:13-21

40. Hakkinen JP, Miller MW, Smith AH et al (1995) Measurement of organ blood flow with coloured microspheres in the rat. Cardiovasc Res 29:74-79

41. Itoh T, Satou T, Nishida S et al (2009) Expression of amyloid precursor protein after rat traumatic brain injury. Neurol Res 31:103-109

\section{Submit your manuscript to a SpringerOpen ${ }^{\circ}$ journal and benefit from:}

- Convenient online submission

- Rigorous peer review

- Open access: articles freely available online

High visibility within the field

- Retaining the copyright to your article

Submit your next manuscript at $\boldsymbol{\nabla}$ springeropen.com 